\title{
Neanderthals: Species or Subspecies?
}

\author{
Jennah L.A. Clarke
}

\begin{abstract}
This article focuses on the ongoing debate regarding whether Neanderthals should be classified as a subspecies of Homo sapiens or as an entirely separate species. Several lines of evidence are explored, including recent genetic evidence, which suggest that despite rare interbreeding events, Neanderthals should in fact be classified as a separate species.
\end{abstract}

A ccording to Richard Klein (2009, 1), species are defined as individuals assigned together based on appearance and membership in the same procreative unit, while subspecies are defined as morphologically and geographically distinct breeding populations within a species that retain the ability to exchange genes and produce fertile offspring. While this may sound simple and straightforward, these concepts actually contain discrete definitions, whereas the organisms that are characterized by them often do not evolve on a continuous trajectory. The debate regarding whether or not the Neanderthals should be classified as a distinct species from anatomically modern humans has been ongoing for over a century. Several researchers have attempted to solve this evolutionary mystery; however, they continue to remain divided on the issue today (Klein 2009; Márquez at al. 2014; Weaver 2003, 2009; Wolpoff et al. 2004).

While it has recently been shown that all non-Africans contain a small percent of $\mathrm{Ne}$ anderthal DNA, whether or not they produced enough fertile offspring to constitute membership within the same species is questionable. It is extremely difficult to determine where a species or subspecies begins and ends, and at what point they are no longer biologically compatible. Matters are further complicated when one of the proposed species in question is extinct, in which case specimens must be studied rigorously in order to reach some sort of hypothesis or conclusion. Fortunately, the Neanderthals are one of the most-studied hominins due to the wealth of relevant archaeological material that has been uncovered, and to the cold conditions in which they lived that preserved their DNA.

Based on fossil and archaeological evidence, it had been widely speculated that $\mathrm{Ne}$ anderthals were simply extreme versions of cold-adapted humans that were capable of the same level of cognitive and symbolic thought as anatomically modern Homo sapiens (Harvati 2003; Hublin 2014; Klein 2009; Márquez at al. 2014; Pearce et al. 2013; Weaver 2003, 2009; Wolpoff at al. 2004). While this sounds theoretically plausible, ultimate classification is far more complex in that it must satisfy certain criteria that truly constitute a species versus a subspecies. Up until very recently, Neanderthals had met all but one criterion for the delineation as a separate species as set out by Osbjorn Pearson (2008). Today, with the help of recent genomic data (Castellano et al. 2014; Neves and Serva 2012; Prüfer et al. 2014; Sankararaman et al. 2014; Vernot and Akey 2014) it could be argued that this distinct classification is in fact warranted.

According to Pearson $(2008,44)$, biological approaches view speciation as a process during which various criteria for what constitutes a "species" differentiate the members of an evolving lineage from its closest living relatives. The purpose of this paper is to provide evidence that satisfies each criterion outlined by Pearson, and to suggest that by the time anatomically modern humans were spreading out of Africa, the Nean-

Undergraduate student; Department of Anthropology, University of Alberta Author contact: jclarke2@ualberta.ca 
derthals had nearly reached full speciation. His list of criteria contain the following: divergence of populations and geographic separation, establishment of identifiable morphology, development of different apomorphies, selection against hybrid individuals, establishment of differing mate recognition systems, reproductive isolation, and ultimately, a complete lack of viability of hybrids (Pearson 2008, 44). Based on several anatomical and genetic studies (Castellano et al. 2014; Currant and Excoffier 2011; Harvati 2003, Hublin 2014; Márquez et al. 2014; Neves and Serva 2012; Pearce et al. 2013; Pearson 2008, Prüfer et al. 2014; Rak et al. 2002; Ramirez 2004; Sankararaman et al. 2014; Vernot and Akey 2014; Weaver 2003, 2009; Wolpoff at al. 2004), it can be demonstrated that the Neanderthals had adapted and evolved separately in Eurasia to the extent that their morphology and genes were no longer compatible with their ancestors who were simultaneously evolving in Africa.

\section{Divergence of Populations And Geographic Separation}

Researchers have long debated the origin of anatomically modern humans based on two models: Multiregional Continuity and Out of Africa (Klein 2009). The Multiregional Continuity model assumes that archaic populations, including Neanderthals, inhabited all regions of the Old World, constantly exchanged genes, and eventually reached their current modern state, whereas the Out of Africa model posits that one single ancestral anatomically modern population evolved in Africa approximately $200 \mathrm{ka}$, migrated out, and replaced the contemporary archaic populations of Europe and Asia. A recent publication on the Neanderthal genome by Kay Prufer et al. (2014) have definitively ruled out the Multiregional Continuity model based on genetic differences between Neanderthals, Denisovans, and present-day humans. They estimate that the population split time between modern humans and Neanderthals occurred somewhere between 550-765 ka (Prufer at al. 2014, 45). This is consistent with the fossil record, which indicates that some members of Homo heidelbergensis spread into Europe from Africa within this time range and began to develop more Neanderthal-like traits, while those that remained in Africa simultaneously developed more anatomically modern traits.

Prufer et al. (2014, 45) also estimated the population split time between Neanderthals and Denisovans to be somewhere between 381-473 ka; however, their gene exchange was particularly low, which may be due to the fact that Denisovans were geographically isolated as far as the Altai Mountains in Siberia. The potential geographic divergence of Neanderthals is further highlighted in a study based on epigenetic maps. Jean-Jacques Hublin $(2014,1339)$ concluded that Neanderthal populations were small and isolated from each other, which combined with low genetic diversity favoured conditions for rapid and random fixation of features by genetic drift. This is also in agreement with the fossil record, whereby $H$. heidelbergensis appears to have evolved into Neanderthals in Europe at a much quicker rate $(550 \mathrm{ka})$ than it did into anatomically modern humans in Africa (200 ka). Hublin (2014, 1339) suggested that rapid divergences and low genetic diversity indicate that two different evolutionary pathways were at work in Neanderthals and anatomically modern humans, which could be responsible for their marked differences in morphology. These factors may also explain why Neanderthals and Denisovans diverged relatively quickly, although we have yet to discover Denisovan hominin remains with which to compare differences in their morphologies.

\section{Establishment of Identifiable Morphology}

Neanderthals exhibit a wide range of traits, both cranially and post-cranially, that distinguish them from contemporary hominins, particularly anatomically modern humans. An initial explanation by Timothy Weaver (2003) focused on adaptation to the cold glacial climate in Europe as the driving mechanism of their identifiable morphology. Weaver $(2003,6926)$ attributed many postcranial differences to cold-adaptation, including the fact that Neanderthals had wide bodies and short, stocky limbs, which would have promoted low surface area to mass and enhanced heat retention in the cold climate. He also studied the femoral head and its articulations and noticed 
that the thick, rounded shafts were accompanied by widening pelvic apertures and more flared iliac blades (Weaver 2003, 6928). When compared to present-day humans, Weaver (2003, 6926) concluded that these features, combined with overall thick bone density, indicated that Neanderthal morphology was hyperpolar or hyperarctic due to their extreme body proportions that exceeded those of modern-day Inuit peoples.

A later study by Weaver $(2009,16032)$ based on geometric-morphometrics suggested that Neanderthal morphology was more likely due to genetic drift with respect to their cranio-facial features. Milford Wolpoff et al. (2004, 531) also elaborated on identifiable Neanderthal cranial features that included retromolar space, taurodontism, supra-orbital torus, receding forehead, occipital bun, suprainiac fossa, projecting mid-face, large nasal cavity, and lack of mental eminence. Both authors concur that these features did not show up all at once, and that they gradually accumulated over an approximate 300,000-year period. Regardless of the mechanism by which these traits were accumulated, Neanderthals had clearly diverged from their predecessor $H$. heidelbergensis and were measurably distinguishable from contemporary anatomically modern humans.

Katerina Harvati (2003) also highlighted the extent of the divergence of Neanderthal features using a measure of both intra- and inter-specific variation. She measured the differing cranio-facial features mentioned above and compared their distances from anatomically modern humans to the distances among present-day human populations, as well as to the distances between the two commonly recognized chimpanzee species Pan troglodytes and Pan paniscus. She discovered that the morphological distances between Neanderthals and anatomically modern humans overall were consistently greater than the distances among present-day human populations and greater than the distances between the two chimpanzee species (Harvati 2003, 109). Harvati $(2003,126)$ used these findings to conclude that the Neanderthal's great morphological and genetic distance would most likely have prevented them from contributing to modern humans and should therefore be classified as a different species. Although her argument is compelling, it would require the additional satisfaction of the following criteria discussed in order to support this claim.

\section{Development of Different Apomorphies}

Due to the small and isolated populations of Neanderthals in glacial Europe, genetic drift likely took hold and fixed certain apomorphies that could distinguish them from other species. One recent area of study has focused on the nasal complex of Neanderthals. By comparing them to those of modern humans, Marquez et al. (2014, 2121) argued that Neanderthal adaptation to cold climates could have necessitated other adaptations such as bony medial projections at the piriform aperture rim and mid-facial prognathism. The authors suggest that the evidence for this defining apomorphic trait lies in its presence among juvenile specimens, which indicates that Neanderthals developed these swellings early in their ontogeny, while modern humans never exhibit this peculiar nasal anatomy at any stage of development (Marquez at al. 2014, 2133). Taken together, this strongly suggests that their growth trajectories are distinct and that these groups therefore represent different species.

Yoel Rak et al. (2002) discovered an apomorphy early on by quantifying the curve of the mandibular notch and comparing it to that of $H$. erectus, early $H$. sapiens, and present-day anatomically modern humans. The results indicated that Neanderthals differ more in ramus morphology to $H$. sapiens than do $H$. sapiens to H. erectus (Rak et al. 2002, 199). The authors concluded that this deviation in Neanderthals must imply a profound specialization of the masticatory system and significant repercussions regarding the size of their gape (Rak et al. 2002, 202). This unique morphology certainly constitutes a diagnostic feature and warrants yet another indication of a completely separate growth trajectory than that of contemporary anatomically modern humans. It also reinforces the notion of a single African ancestry for anatomically modern humans who are probably closer morphologically and genetically to the African $H$. erectus and $H$. heidelbergensis populations that never left Africa. 
Life history traits correlate closely with dental enamel formation and growth, which allow us to determine the developmental shifts that warrant species-level distinctions. Fernando Ramirez Rossi and José Bermudez de Castro (2004) measured the differences in enamel formation between Neanderthals, modern H. sapiens, $H$. heidelbergensis, and the alleged $H$. antecessor. The results indicated surprisingly rapid development in Neanderthals due to having the shortest period of growth (Ramirez Rossi and Bermudez da Castro 2004, 936). The authors concluded that since Neanderthals developed even faster than their predecessor $H$. heidelbergensis despite having a larger brain, this represented an apomorphy in growth and an evolutionary reversal that warranted distinction as a separate species (Ramirez Rossi and Bermudez de Castro 2004, 938). This, combined with differences in brain development as mentioned below, is a strong indicator that Neanderthals would not be biologically compatible with anatomically modern humans. It is hard to imagine how a hybrid would be affected by such profound differences in development and growth between the parents.

Important developmental apomorphies have also been recognized in Neanderthal brain organization. Their unusually large brains have often been interpreted as comparable to that of anatomically modern humans, and thus similar in cognitive and symbolic capacities; however, studies have shown that their elongated brain cases are most likely indicative of differing lobe processes. Eiluned Pearce et al. (2013, 1-4) observed that Neanderthals show lateral widening, but overall flattening of the parietal lobes that are responsible for cognition, as well as a relatively larger occipital lobe that is responsible for visual processing. The authors suggested that this, combined with larger eye orbits and high lean body mass, is indicative of their apomorphic brain development, including larger visual cortices and more neural tissue invested in somatic areas that are involved in body maintenance and control (Pearce et al. 2013, 5). Thus, Neanderthals would not have been able to think and process the way that early anatomically modern $H$. sapiens $\mathrm{did}$, and they most certainly would not have been able to think and process the way that humans do today. This, combined with other developmental apomorphies may suggest that a separate species is warranted for Neanderthals.

\section{Selection Against Hybrid Individuals}

Recent studies on the Neanderthal genome have contributed breakthroughs to our understanding of the origins of anatomically modern humans, as well as to the question of potential interbreeding and Neanderthal contribution to modern human DNA. While Prufer et al. (2014, 45) reported that Neanderthals contributed 1.5$2.1 \%$ of their DNA to all non-Africans, this should not be interpreted as definitive evidence in support of subspecies classification. According to Sriram Sankararaman et al. $(2014,355)$, it was originally estimated that initial interbreeding episodes could have been as high as 62\% in East Asian populations and 64\% in European populations based on a genetic map of 1,004 presentday humans; however, widespread negative selection against Neanderthal ancestry was detected shortly afterwards. The authors discovered that the largest deserts of Neanderthal ancestry were on the X-chromosome, which was indicative of reduced male fertility of hybrids and an introgression success rate of only 3\% (Sankararaman et al. 2014, 356). They concluded that the introduction of Neanderthal-derived deleterious alleles in hybrids could explain why anatomically modern humans were more resistant to Neanderthal ancestry, and why the effect of negative selection resulted in a 'quantitatively large' reduction of introgression based on the estimation of initial interbreeding episodes mentioned above (Sankararaman et al. 2014, 356). This widespread selection against hybrids could be interpreted as evidence in support of the potential biological incompatibility of these two species.

Prufer at al. $(2014,48)$ also suggested that negative selection of Neanderthal ancestry could have contributed to their widespread population decrease after anatomically modern humans spread out of Africa. It remains a mystery as to why modern human DNA was not detected in the Neanderthal genome; it appears as though they contributed DNA to us, but we did not con- 
tribute any to them. Once again, this may have been due to the negative selection of sterile hybrids, seeing as a rapid decrease in population would have severely impacted the number of potential male mates for future female Neanderthals. Benjamin Vernot and Joshua Akey (2014, 1021) reported similar data, which suggested that the DNA sequence divergence between modern humans and Neanderthals was a barrier to gene flow in some regions of the genome and was associated with deleterious fitness consequences. However, they also concluded that modern humans were relatively lucky in that advantageous adaptive Neanderthal genes such as lighter skin pigmentation and immunity to diseases were positively selected at a relatively rapid rate (Vernot and Akey 2014, 1021). These traits would have allowed anatomically modern humans spreading out of Africa to successfully adapt to the colder climate in Europe, whereas potentially sterile hybrids would have had difficulty reproducing and adapting overall.

\section{Establishment of Differing Mate Recognition Systems}

It has been widely suggested that Neanderthals maintained smaller and more isolated populations, which would have promoted lots of interbreeding with close relatives. Prufer at al. (2014, 45) have supported this assumption with their findings that the parents of a Neanderthal woman from the Altai Mountains from whom they extracted a genome sequence were related at the level of half-siblings and that mating among close relatives was common amongst her recent ancestors. This ultimately had implications for how and why the Neanderthal populations appeared to diverge relatively quickly due to their limited gene pool and potential for genetic drift. These long runs of homozygosity were also present in three other late Neanderthal sequences reported by Sergi Castellano et al. (2014, 6667), which indicated that mating with relatives was much more common in Neanderthals than it is in present-day human populations. They also concluded that Neanderthal genetic diversity within continents differed more than present-day human genetic diversity does between continents
(Castellano et al. 2014, 6667). Based on this information, it seems as though the Neanderthals maintained a highly localized form of endogamy, which may have contributed to their rapid divergence and limited biological compatibility with modern humans. This finding also has implications for the reproductive isolation section below.

\section{REPRODUCTIVE ISOLATION}

In contrast to Sankararaman et al. (2014), Mathias Currant and Laurent Excoffier (2011, 15129) hypothesized that the very low rate of interbreeding could imply the existence of 'extremely strong barriers' to gene flow between the two species due to a very low fitness of hybrids, strong avoidance of interspecific mating, or both. Under most demographics within their models, the interbreeding success rate was found to be below $2 \%$, which they suggest might actually be inflated due to recurrent admixture at the wave front where the local invading population (anatomically modern humans) was still growing, and thus passing on the introgressed genes (Sankararaman 2011, 15132). Currant and Excoffier $(2011,15132)$ also state that uneven introgression from the local to the invading species naturally occurs during range expansion, and that genes introgressing in the invading population are amplified by population growth, which could have a very large final impact.

These findings could explain why anatomically modern humans spreading out of Africa successfully adapted to the new climate in Europe by quickly acquiring these advantageous Neanderthal traits that are still expressed in nonAfricans today, and passing them onto their offspring over several generations. This observable large final impact on non-Africans is deceiving based on the authors' estimation that successful interbreeding events would have only occurred once every 23-50 years over a 10,000 year range expansion of modern humans (Sankararaman 2011, 15132). The authors conclude that these interbreeding events were 'extremely rare', which suggests that although interbreeding did take place, it occurred so rarely that it may not be strong enough evidence to constitute membership within the same species. 
Armando Neves and Maurizio Serva (2012, 8) apply a different approach to the extreme rarity of interbreeding events between Neanderthals and anatomically modern humans emerging out of Africa by estimating that a single pair of individuals interbred at a 77-generation interval over 130,000 years of co-existence. They also posit that these breeding events would have occurred in breeding zones where early anatomical modern humans were leaving Africa into the Middle East and Western Europe (Neves and Serva 2012, 8). According to this estimate, the biological and genetic barriers to reproductive success between the two groups were so strong that although they co-existed side-by-side for so long they barely exchanged genes. Despite the differing analogies and potential mating success proposed by these studies, each have shown that only $2 \%$ of gene flow could have occurred from Neanderthals into all non-African modern humans, which is exactly what Prufer et al. (2014) reported.

\section{LACK OF Viability of OfFSPRING}

As mentioned above, Castellano et al. (2014) observed that each of the three Neanderthal individuals from whom they obtained their genome sequences carried a larger fraction of putatively deleterious alleles than present-day humans. The authors attributed this to the population decrease that occurred, which over a long period of time would reduce the efficacy of purifying selection in Neanderthals and contribute to an even larger amount of deleterious alleles (Castellano et al. 2014, 6667). These deleterious alleles most likely contributed to the sterility of male hybrids produced by these two species as well. Based on the lack of viable hybrid offspring, it would appear that the Neanderthals and anatomically modern humans continued to evolve on different trajectories and eventually became biologically incompatible with each other. Although they contributed a small amount of their genes to non-Africans, which resulted in a modest amount of phenotypic and medical variations, they did not contribute to our overall ancestry as a species, which includes all Africans as well.

\section{ConCLUSION}

Due to the limited compatibility of Neanderthals and anatomically modern humans demonstrated by these studies, and the fact that Neanderthals went extinct in their own environment, they may very well have been "evolutionary dead ends" after all. There will always be subspecies and admixture at some level, and unfortunately, genome sequences are not always a feasible option for decipherment. Furthermore, it has also been demonstrated that some hominin groups that allegedly belong to the same population are incredibly diverse to the extent that if discovered in isolation, they would be assigned to multiple species (Arsuaga et al. 2014; Lordkipanidze et al. 2013). Since the fossil record is often all that we have, it will continue to be up to our imaginations to reconstruct the past to the best of our ability.

After over a century of research, the Neanderthal species-versus-subspecies mystery may very well be solved as more ancient genomic data is recovered and studied, but by the same token, the debates may just be getting started. As more hominin and archaeological remains are uncovered and relevant technologies advance, we are constantly forced to ask new questions and rearrange what we thought we already knew about our distant relatives and ancestors. While progress appears to have been made, the difficulty in species classification appears to stem from our limited understanding of what it means to be a species overall, especially in light of genetics, which we do not yet fully comprehend. Despite recent breakthroughs in this field, the issue regarding hominin species classification based on fossil and genomic data (if at all) remains unresolved. However, in the balance of the data presently available, including morphological and developmental apomorphies, an overall derived evolutionary trajectory, and the apparent unviability of hybrid offspring, a solid case can be made for a distinct species classification for Homo neanderthalensis.

\section{ACKNOWLedgements}

This article is a term paper that was written for ANTH 391: Hominid Evolution with Dr. Pamela Willoughby. 


\section{References Cited}

Arsuaga, J.L., I. Martinez, L.J. Arnold, A. Aranburu, A. Garcia-Tellez, W.D. Sharp, R.M. Quam, C. Falgueres, A. Pantoja-Perez, J. Bischoff, E. Poza-Rey, J.M. Pares, J.M. Carretero, M. Demuro, C. Lorenzo, N. Sala, M. Martinon-Torres, N. Garcia, A. Aleazar de Velasco, G. Cuenca-Bescos, A. GomezOlivencia, D. Moreno, A. Pablos, C-C. Shen, L. Rodriguez, A.I. Ortega, R. Garcia, A. Bonmati, J.M. Bermudez de Castro, and E. Carbonell. 2014. "Neandertal roots: Cranial and chronological evidence from Sima de los Huesos." Science 344(6190): 1358-1363.

Castellano, Sergi, Genis Parra, Federico A. Sanchez-Quinto, Fernando Racimo, Martin Kircher, Susanna Sawyer, Qiaomei Fu, Anja Heinze, Bigrit Nickel, Jesse Dabney, Michael Siebauer, Louise White, Hernan A. Burbano, Gabriel Renaud, Udo Stenzel, Carles lalueza-Fox, Marco de la Rasilla, Antonio Rosas, Pavao Rudan, Dejana Brajkovic, Zeljko Kucan, Ivan Gusic Michael V. Shunkov, Anatoli P. Derevianko, Bence VIola, Matthias Meyer, Janet Kelso, Aida M. Andres, and Svante Paabo. 2014. "Patterns of coding variation in the complete exomes of three Neandertals." Proceedings of the National Academy of Sciences 111(18): 6666-6671.

Currant, Mathias, and Laurent Excoffier. 2011. "Strong reproductive isolation between humans and Neanderthals inferred from observed patterns of introgression." Proceedings of the National Academy of Sciences 108(37): 15129-15134.

Harvati, Katerina. 2003. "The Neanderthal taxonomic position: models of intra- and interspecific craniofacial variation." Journal of $\mathrm{Hu}$ man Evolution 44(1): 107-132.

Hublin, Jean-Jacques. 2014. "How to build a Neandertal.” Science 344(6190): 1338-1339.

Klein, Richard G. 2009. The Human Career: Human, Biological, and Cultural Origins, 3rd edition. Chicago: University of Chicago Press. Lordkipanidze, David, Marcia S. Ponce dE Leon, Ann Margvelashvili, Yoel Rak, G. Philip RIghtmire, Abesalom Vekua, Chris-
Toph P. E. Zollikofer. 2013. "A Complete Skull From Dmanisi, Georgia, and the Evolutionary Biology of Early Homo." Science 342(326) 326-331.

Márquez, S., Anthony S. Pagano, Eric Delson, William Lawson, and JefFrey T. Laitman. 2014. "The Nasal Complex of Neanderthals: An Entry Portal to their Place in Human Ancestry." The Anatomical Record 297(11): 21212137.

Neves, Armando G.M. and Maurizio Serva. 2012. "Extremely rare interbreeding events can explain Neanderthal DNA in living humans." PLoS One 7(10): 1-10.

Pearce, Eiluned, Chris Stringer, and R.I.M. DunBar. 2013. "New insights into differences in brain organization between Neanderthals and anatomically modern humans." Proceedings of the Royal Society B 280(20130168): 1-7.

Pearson, Osbjorn M. 2008. "Statistical and biological definitions of 'anatomically modern' humans: Suggestions for a unified approach to modern morphology." Evolutionary Anthropology 17(1): 38-48.

Prüfer, Kay, Fernando Racimo, Nick PatterSOn, Flora Jay, Sriram Sankararaman, Susanna Sawyer, Anja Heinze, Gabriel Renaud, Peter H. Sudmant, Cesare de Filippo, Heng Li, Swapan Mallick, Michael Dannemann, Qiaomei Fu, Martin Kircher, Martin Kuhlwilm, Michael Lachmann, Matthias Meyer, Matthias Ongyerth, and Michael Siebauer. 2014. "The complete genome sequence of a Neanderthal from the Altai Mountains." Nature 505(7481): 43-49.

Rak, Yoel, Avishag Ginsburg, and Eli Geffen. 2002. "Does Homo neanderthalensis play a role in modern human ancestry? The mandibular evidence." American Journal of Physical Anthropology 19(3): 199-204.

Ramirez Rossi, Fernando V., And José M. BerMUDEZ DE CASTRO. 2004. "Surprisingly rapid growth in Neanderthals." Nature 428(6986): 936-939.

Sankararaman, Sriram, Swapan Mallick, MiChael Dannemann, Kay Prüfer, Janet KelSo, Svante PäÄвo, Nick Patterson, And DaVID REICH. 2014. "The genomic landscape of 
Neanderthal ancestry in present-day humans." Nature, 507(7492): 354-357.

Vernot, Benjamin, and Joshua M. Akey. 2014.

"Resurrecting surviving Neandertal lineages from modern human genomes." Science 343(6174): 1017- 1021.

Weaver, Timothy D. 2003. "The shape of the

Neandertal femur is primarily the consequence of hyperpolar body form." Proceedings of the National Academy of Sciences 100(12): 6926-6929.

. 2009. "The meaning of Neandertal skeletal morphology." Proceedings of the National Academy of Sciences 106(38): 16028-16033.

Wolpoff, Milford H., Bruce Mannheim, Alan Mann, John Hawks, Rachel Caspari, Karen R. Rosenberg, David W. Frayer George W. Gill, and Geoffrey Clark. 2004. "Why not the Neandertals?" World Archaeology 34(4): 527-546. 